\title{
Design Classes for Hybrid Simulations Involving Agent-Based and System Dynamics Models
}

\author{
Chris Swinerd, Ken R. McNaught ${ }^{\text {s }}$ \\ May, 2011 \\ \$Corresponding author. Tel: +44(0)1793 785288; Fax: +44(0)1793 784196. \\ E-mail addresses: c.swinerd@cranfield.ac.uk (C. Swinerd), k.r.mcnaught@cranfield.ac.uk (K.R. McNaught)
}

Department of Informatics and Systems Engineering, Cranfield University, Defence Academy of the UK, Shrivenham, Swindon SN6 8LA, UK.

\begin{abstract}
Hybrid simulation involves the use of multiple simulation paradigms, and is becoming an increasingly common approach to modelling modern, complex systems. Despite growing interest in its use, little guidance exists for modellers regarding the nature and variety of hybrid simulation models. Here, we concentrate on one particular hybrid - that involving agent-based and system dynamics models. Based on an up-to-date review of the literature, we propose three basic types of hybrid agent-based system dynamics simulations, referred to here as interfaced, integrated and sequential hybrid designs. We speculate that the classification presented may also be useful for other classes of hybrid simulations.
\end{abstract}

Keywords: Simulation; system dynamics; agent-based; complex systems; hybrid modelling

\section{Introduction}

Simulation in its various guises has consistently been reported as one of the most frequently used techniques by practitioners of Operational Research (OR) [19]. Both discrete-event simulation and, more recently, system dynamics are widely taught on OR degree courses. Increasingly, agent-based simulation is also found on many OR course curricula, largely driven by its popularity among modellers of social systems. Increasing familiarity with more than one simulation methodology might help explain the growing interest in hybrid simulation approaches, i.e. approaches which combine at least two of the three methodologies discussed. For example, Jahangirian et al. [22] report increasing use of hybrid simulation approaches within manufacturing simulation. Another explanation might be that the nature of many of the problems which OR analysts are now confronted with calls for a multimethodology or hybrid approach to be taken. The complex, multi-faceted nature of many modern-day systems can pose considerable challenges for traditional, single-methodology simulation approaches. While these challenges are often successfully overcome, they may stretch the methodology and call for considerable ingenuity 
on the part of the modeller. In such cases, it may be that an alternative simulation approach, either using another modelling paradigm or a hybrid approach, could provide a simpler, more natural or more efficient solution.

This paper is not intended to provide a general review of hybrid simulation. Nor does it consider all of the hybrid combinations in equal measure. Instead, it focuses primarily on the class of hybrid simulation involving agentbased simulation and system dynamics, referred to here as AB-SD simulation. Our reasons for focusing on this particular combination are firstly that it is less well researched and understood than the SD-DES combination and secondly that we believe it offers a potentially useful approach to the modelling of complex adaptive systems (CAS). Such systems are defined by the US Argonne National Laboratory [5], a leading exponent of agent-based simulation, as 'fluidly changing collections of distributed interacting components that react to both their environments and to one another'. For example, McCarthy et al. view the development of new products as a complex adaptive system [32]. They state that 'Complex adaptive systems consist of a nested and scaleable system of agents; that is, the level of system abstraction could be an individual, a group, or an organization' [32, $p$ 442]. They go on to say that '...nonlinearity and feedback can occur at multiple levels between individual agents and between groups of agents' [32, p443].

Since nonlinearity and feedback are essential parts of the SD worldview and are explicitly represented within SD models, it is little wonder that SD has often been applied to model CAS. A fine example is provided by Sterman and Wittenberg [51] who developed an SD model to illustrate Kuhn's arguments concerning the rise and fall of academic paradigms. An implicit notion within this model was that each paradigm could be regarded as an agent. This notion of agency can also be observed in several other SD models of CAS. In fact, it is not always clear whether a particular model should be regarded as purely an SD model or as a hybrid AB-SD model. Perhaps what Duggan [13] refers to as agent-oriented SD models is a more accurate description of such models. It is our belief, however, that some CAS are best represented by truly hybrid AB-SD models. In this paper, we review several such hybrid models and, based on this review and related work by Shanthikumar and Sargent [48], suggest a classification for hybrid AB-SD simulation models. We speculate that this classification might also be useful for hybrid simulation more generally. It is recognised that further research is required to better understand where and when these classes of hybrid model design are most appropriately applied and to what benefit. This review of design classes is intended as a first step towards a structured approach to such research.

\section{Simulation paradigms}

The paradigms of system dynamics (SD) and agent-based $(A B)$ simulation are both well established with much openly published literature available that spans decades of research. They have been widely applied across many domains to predict system behaviour using either deductive SD or inductive $A B$ approaches (also commonly referred to as top-down and bottom-up approaches, respectively). As such, these paradigms represent 
complementary approaches to modelling. Furthermore, each paradigm has its own particular characteristics that make it suitable for modelling certain aspects of systems.

Representing the resources and dynamics within a system as a set of stocks and the flows between them, SD captures feedback and delay processes to model system behaviour over time. The stocks provide aggregate representations of entities within a system, with flows in and out of them regulated by feedback and delay such that resultant system performance can be non-linear and, indeed, sometimes counter-intuitive. Resource flows correspond to the mean rates at which entities within the system change state. The SD approach was founded by Jay Forrester in the 1950s and explained in his seminal text 'Industrial Dynamics' [15].

$A B$ modelling represents system entities as individuals. Referred to as agents, these entities interact with each other and their environment according to rules which are often simple and local in nature and from which higher level system behaviour can be observed. Cellular automata (CA) can be considered as a simple form of $A B$ modelling. CA comprise a grid of cells, each of which can be in one of several discrete states based on simple transition rules. Cells can have a degree of influence on neighbouring cells as required and from this, emergent patterns of behaviour can sometimes be observed. Like $C A, A B$ modelling readily offers the opportunity for spatial dimensions to be represented where they might affect system behaviour.

Facilitated by the advent of object-orientated programming and implemented using asynchronous programming techniques, agent interactions are defined by a set of decision-making rules and the agents are given autonomy to interact with each other and the environment such that temporal and spatial macro behaviours (aggregated behaviour at a level higher than that of the rules allocated to agents) can be observed. However, as Garro and Russo [16] point out, despite the increasing range of domains which AB simulation can be applied to, experts in those domains with limited programming expertise often face great challenges in attempting to utilise such an approach.

Comparisons of the various simulation methodologies have been made from time to time. Schieritz and Milling [44] provide a useful comparison of SD and $A B$ in an aptly titled paper which sums up the distinction, 'Modeling the forest or modeling the trees'. Exploring differences between their preferred simulation paradigms, Morecroft and Robinson [34] considered the same problem regarding fish stocks from both SD and DES perspectives, respectively. Widening the number of expert modellers involved, Tako and Robinson [52] investigated how DES and SD modellers went about conceptualising and developing models of the same problem regarding prison populations within the two paradigms. Lorenz and Jost [27] compare all three methodologies, discussing their underlying assumptions and technical differences. They emphasise the importance of asking what the purpose of the final model is in addition to accounting for the nature of the system being modelled. 
It is certainly the case that many systems can be modelled in equivalent ways by different simulation paradigms. North and Macal [36] showed that entirely equivalent representations (numerically equivalent to 12 significant figures) of the well-known Beer Game Simulation [50] could be developed using either an SD approach or an AB approach. Macal [28] notes that such models are an interesting subset of SD models and inform model developers that alternate approaches may be available to achieve exactly the same results. However, in many cases, depending on developer experience and the intended use of the model, one paradigm will present a more natural choice. Furthermore, while it may often be possible to model a system using a single paradigm approach in isolation, a hybrid approach might be preferred for reasons of efficiency or transparency. The overall effort involved in 'stretching' a particular paradigm to represent key aspects of the system being modelled has to be balanced against the overall effort involved in employing a hybrid approach.

Focusing on those OR models which hybridise simulation with an analytic method, Shanthikumar and Sargent [48] and, in a later review, Sargent [42], proposed a classification of hybrid models by outlining, with examples, four classes of hybrid model. They defined analytic models as 'a set of equations that characterise a system or a problem entity. Its solution procedure usually uses either an analytical equation or a numerical algorithm that has been developed for the set of model equations to obtain the desired results', while simulation models were defined as "dynamic or operating models of a system or problem entity that "mimics" the operating behaviour of the system or problem entity and contains its functional relationships' [48, p1030].

Sargent [42] looked to assess the impact of his earlier work with Shanthikumar by comparing the work reported on hybrid modelling by the wider research community before and after its publication. He concluded that despite significant advances in computer hardware and software systems, there was little evidence of real progress in adopting hybrid models (or modelling). He believed that in the main this was due to the little attention given over to this approach to analysis in textbooks or the classroom, where the various techniques tended to be considered in isolation.

A number of articles published since Sargent's review, confirm his views. For example, in describing the correspondence between modelling paradigms, Lane [24, p118] states 'If we seek to operate in the real world, it is surely better to have available a range of approaches and tools that draw on the breadth of systems thinking.' Five years on, Phelan [38] notes observations on the correspondence between what he refers to as complexity and systems theory. Two years later, Scholl responded to these observations and discussed in his two papers [46, 47] the potential for combining agent-based and system dynamics paradigms, calling for cross study and joint research. Whilst by 2004 there is increasing evidence of hybrid AB-SD approaches being adopted, Borshchev and Filippov [7] reflect on the ongoing segregation in the teaching of modelling techniques that has the tendency to encourage separate practitioner communities. One can also reflect on the tendency for most software packages, conferences, societies and journals to focus on specific paradigms of modelling and simulation. 
Following an overview in section 3 of the hybrid model classes proposed by Shanthikumar and Sargent [48] and in section 4 a discussion of system decomposition to support such modelling approaches, section 5 comprises three sub-sections, each of which considers a particular hybrid design. Each sub-section is informed by simulation work reported from a diverse range of application areas in order to establish the archetypes as credible representations of hybrid simulation modelling. Before some concluding remarks, a penultimate section considers the representation of system hierarchy (or scale) and introduces concepts for models to better reflect the 'nature' of a system as a function of time or events.

\section{A Unifying View and Some Definitions}

This section introduces the terminology used throughout the rest of the paper and provides an overview of the four hybrid model classes proposed by Shanthikumar and Sargent [48]. An important observation which they make is that while simulation models permit a greater degree of realism than analytic models, the cost of model development and use is much cheaper for analytic models than simulation models. A key motivation for investigating hybrid design concepts, then, is to deliver cost-effective and computationally efficient solutions.

Their original four classes of hybrid model are described as follows [48, pp1034-1035]:

1. Class I - 'a model whose behaviour over time is obtained by alternating between independent analytic and simulations models';

2. Class II - 'a model in which an analytic and simulation model operate in parallel over time and with interactions between them';

3. Class III - 'a model in which a simulation model operates in a subroutine way for an analytic model of the total system'; and

4. Class IV - 'a model in which a simulation model is used to model the total system but which requires values for a portion of the system or input parameters, from an analytic model'.

They go on to propose that if the time-dependent behaviour of the system can be completely decomposed so that some part of it can be solved analytically then, on the basis of efficiency, one would choose a class I hybrid model. If both model types are required to operate in parallel with respect to time and with interactions between them, then one would use a class II hybrid model. Where complete decomposition is not possible, then again, on the basis of efficiency, one would prefer to use a class III model rather than a class IV model because the analytic model is in general cheaper and computationally more efficient. These model classes are reproduced in Figure 1.

Based on our review of published work covering a considerable range of application areas, we propose that for hybrid AB-SD simulation modelling, the four classes defined above can be reduced to three and that these can be given meaningful, descriptive titles. Such titles convey greater meaning and better infer the nature and utility of the hybrid design concept. 
In assessing the four classes defined by Shanthikumar and Sargent, it can be seen that in the case of class I, the output from analytic and simulation models are 'interfaced'. Here, we suggest that the word 'interface' can be used to describe a point of interaction or communication between elements of different simulation models. When these simulation models are from different paradigms, then we have an interfaced hybrid simulation design.

Where it is possible to decompose sub-systems, or system hierarchy (or scale), through appropriate and efficient use of modelling techniques but where the techniques need to be run in parallel and include feedback between them, as with class II, it is suggested that this design involves more integration of the techniques than the other classes. The word 'integrate' can be used to describe the combining or adding of parts to make a unified whole. It is unclear as to why Shanthikumar and Sargent used dotted lines to represent feedback between models within class II as without this feedback the model approaches class I. It is assumed here that at least one

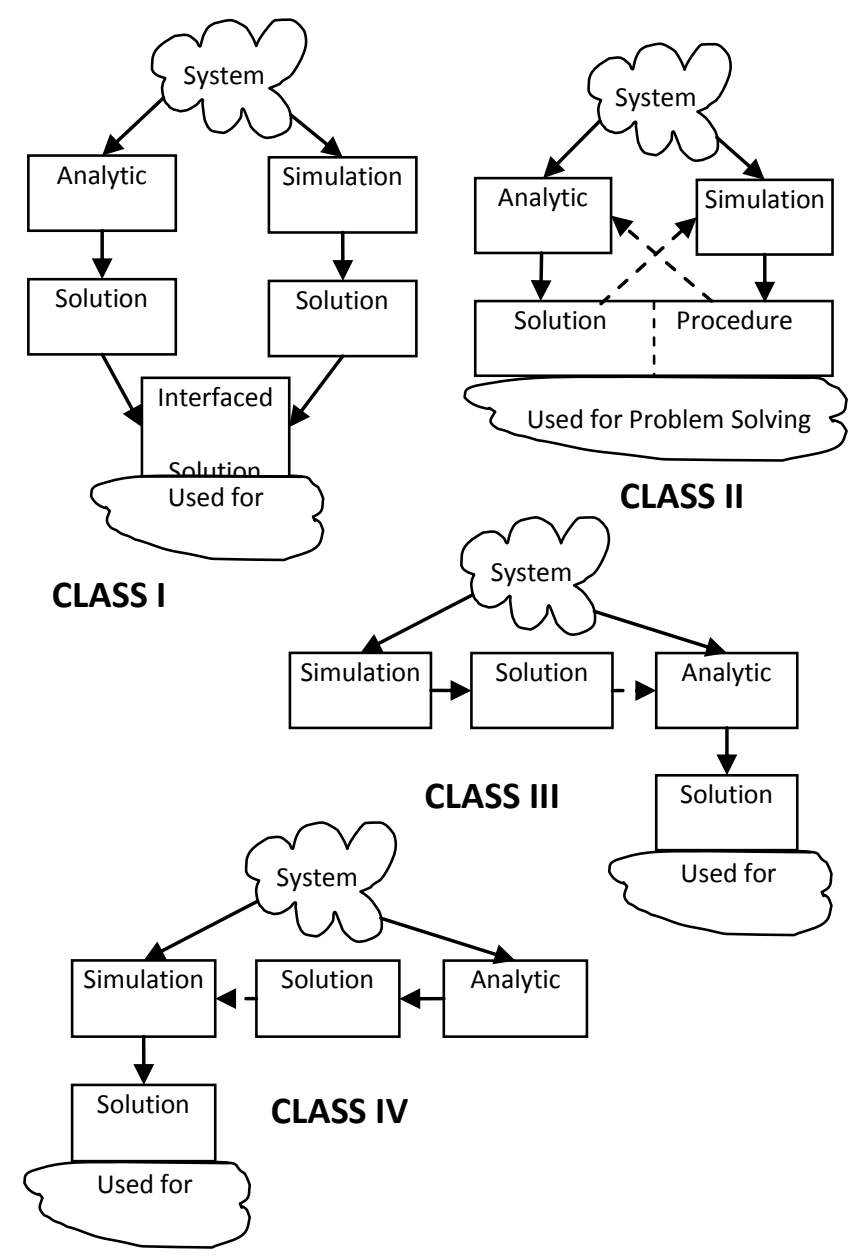

Fig.1. Shanthikumar and Sargent's four classes of hybrid model. Reprinted by permission. Copyright [1983] INFORMS. [Figures 1a, 1b, 1c, 1d from: Shanthikumar, J. G., R. G. Sargent. 1983. A unifying view of hybrid simulation/analytic models and modeling. Operations Research 31(6) 1030-1052.], the Institute for Operations Research and the Management Sciences, 7240 Parkway Drive, Suite 300, Hanover, Maryland 21076, USA. 
of these feedback paths has to exist. The inclusion of feedback reinforces the concept of an integrated design. We define an integrated hybrid simulation as one which contains sustained feedback (not just at one point in time) between simulation models from different paradigms.

Finally, what Shantikumar and Sargent defined as classes III and IV both represent 'sequential' designs, whichever way around one type of model is used to inform the other. The word 'sequence' can be used to describe a logical order of events or processes. We define the use of one simulation paradigm strictly before the use of another to be a sequential hybrid simulation. The first simulation must be capable of producing the required input for the second simulation and then terminating before the second simulation begins.

In reporting on their hybrid AB-SD model applied to the domain of financial stability, Martinez-Moyano et al. [31] describe three types of interaction between modules, relating to different modes of usage of the hybrid model: 'scenario exploration', where the domain $A B$ model is run first and results are sent to the SD model (aligning to the sequential (Classes III or IV) design) ; 'intertwined models', where the domain AB and SD models alternate and potentially pass information between them (aligning to an integrated (Class II) design); and 'crisis response', where the domain $A B$ model is run first on empirical input data and results are then passed on to the SD model (again aligning to the sequential (Class III or IV) design). It is also worth noting their comment [31, p4] that 'In considering the integration of different levels of aggregation in a model, the division of labor between domain $A B$ models and system dynamics models is a major design decision.'

For consistency, the term 'module' is used in the remainder of the paper to describe a modelling component implemented using a particular modelling paradigm. A hybrid simulation is considered to comprise at least two modules from different paradigms. Whilst SD and $A B$ paradigms are the focus of this paper, we believe that the definitions above for describing a hybrid simulation are more generally applicable and can involve any appropriate simulation paradigm. Some diagrammatic examples are provided in section 6 to include DES and CA modules as well as $S D$ and $A B$ modules to reinforce this notion.

\section{Decomposing a System in Order to Establish the Most Appropriate Design}

This section discusses various aspects of decomposing and managing the decomposition of systems so that hybrid models might be utilised to better represent them. Firstly, representation of scales of the system, such as individual, local, regional or national levels, are discussed, including consideration of what is observable through behaviour or describable through equations. The coupling between different scales of the system is potentially one important aspect where hybrid simulations may be shown to add value over and above single paradigm approaches. This is introduced here and discussed further in the subsequent review of reported literature, with examples of socio-economic and socio-ecological coupling covering local and national scales. The need to consider the management of units and time within the hybrid modelling context is examined next before the section concludes with a review of the representation of agents.

\subsection{System Scale}


A key aspect of Shanthikumar and Sargent's work [48] concerns the decomposition of a system in such a way that efficient modelling architectures can be designed which represent system behaviour appropriately.

Lane and Husemann [25], in exploring positive feedback, discuss the relationship between social structures and norms and individual (agent) behaviours (pages 54-55). They illustrate this decomposed feedback process which is simplified here for reference but which illustrates the concept of cross-scale coupling.

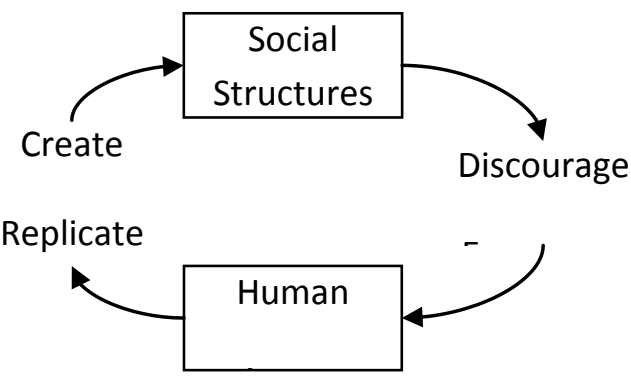

Fig.2. Interplay between acts of human behaviour and social structures [25, p55]. Reprinted by permission. Copyright [2008] John Wiley \& Sons, Ltd. Lane DC and Husemann E (2008). Steering without Circe: attending to reinforcing loops in social systems. System Dynamics Review 24, 37-61.

As concluded by Parunak, Savit and Riolo [37, p15]: 'our ability to adopt the best modelling approach for a given problem depends on developing a collection of cases that demonstrate the respective strengths and weaknesses of the two [AB and SD] approaches'. In their work, Parunak et al. [37] look to reconstruct their $A B$ model representation of a supply chain with an SD representation. In describing the relative merits of each with regard to practical considerations for model structure, they observe that 'AB models are better suited to domains where the natural unit of decomposition is the individual rather than the observable or the equation'. By 'observable', we take this to mean a measurable quantity such as a sum of money or the size of a population - a stock or level in SD modelling parlance. They reinforce this by going on to state that 'equation based models may be better suited to domains where the natural unit of decomposition is the observable or equation rather than the individual'. They also state that 'equation based models (SD models) begin with a set of equations that express relationships among observables' and that $A B$ models begin 'with behaviours through which individuals interact with one another' $[37, \mathrm{p} 12]$. Furthermore, they describe critical relationships that differentiate the approaches such as:

- Individuals are characterised by observables, and affect the values of these observables by their actions;

- Observables are related to one another by equations; and

- Individuals interact with one another through their behaviours.

They illustrate these relationships, as reproduced in Figure 3. 


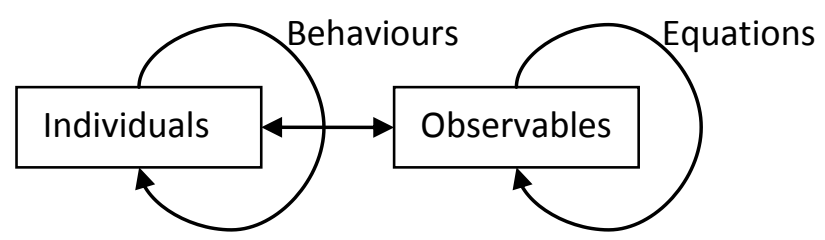

Fig.3. Unifying multiplicities between individuals and observables of a system from Parunak et al. [37]. With kind permission from Springer Science+Business Media: Agent-Based Modelling vs Equation-Based Modelling: A Case Study and Users' Guide, Proceedings of Workshop on Modeling Agent Based Systems, Paris, 1998, Parunak HV, Savit R, and Riolo RL, Figure 13. Copyright [1998] Springer-Verlag.

Confirming the general findings of Parunak, Savit and Riolo, examples from different research domains are summarised below: Wakeland et al. [56], Marin et al. [30], Demirel [11] and Alvarez et al. [3]

Wakeland et al. [56] reported on their investigation into the use of SD and AB modelling for cellular receptor dynamics and their comparative potential for use in education or for planning experiments. While they did not find a simple dividing line indicating when one paradigm or the other would be clearly preferred, they did note that these techniques were complementary, observing that $S D$ is a more natural choice for highly aggregated modelling, while $A B$ is better suited to studying phenomena at the level of individuals. They also concluded that SD models represent the relationships between variables very effectively while agent-based models force more careful consideration of the definition of agents and the specification of their behavioural rules. $A B$ models are better suited to spatial representation and are able to easily portray interactions at the cellular/molecular level.

Marin et al. [30] report on their SD model for workforce planning within NASA's Kennedy Space Centre. Echoing the observations of Shanthikumar and Sargent [48], regarding the preference of analytic models to simulation models on the basis of cost and computational efficiency, they chose to develop their model using SD as they perceived that the complexity associated with other methodologies would be prohibitive. However, in order to enhance the representation of decision-making in their model and especially 'lower-level' decision-making, they propose to incorporate an AB model. This proposal is in line with the findings of Parunak et al. [37] but also indicates the need to represent local conditions (decisions) within the simulation.

Demirel [11] reports on an experiment to replicate modelling of a Supply Chain in both SD (using STELLA [49]) and $A B$ (using NetLogo [35]). A key finding concerns exploring the heterogeneity of agents with respect to their choices and behaviour. Again, this is consistent with the conclusions of Parunak et al. [37]. Similarly, Alvarez et al. [3] recognise the need to capture social tensions within their SD model which represents the broader system aspects arising from the development of the Panama Canal. While they propose to explore neural networks for this purpose, the need to look elsewhere to enhance representation of this social dynamic is worth noting.

In reviewing published material to demonstrate support for Parunak, Savit and Riolo's findings, it was evident that local factors, such as differing social network connectivity or population groupings, for example, may also need to be considered in decomposing a system. The findings of Rahmandad [40], Lorenz and Jost [27] and Chahal and Eldabi [8] also indicate the need to consider this aspect.

Comparing SD with $A B$ modelling for the spread of disease, Rahmandad [40] concludes that $A B$ modelling can better capture the impact of local network structures on the diffusion process. Such local structures can be responsible for non-diffusion, which might not be predicted by more aggregate level modelling such as SD. 
Lorenz and Jost [27] explore factors in choosing modelling paradigms to appropriately represent and analyse complex systems and sub-systems. In working towards an 'orientation framework' in which the purpose, method and object of modelling a system are factored into model selection, their general findings align to the higher level concepts defined by Parunak et al. [37], but also include the need to consider spatial and heterogeneity (that can be considered within 'local conditions') factors. Interestingly, they explore the concept of 'alternative environments' in agent-based modelling with respect to multi-paradigm ( $A B$ and SD) modelling: a 'zero' environment with which agents do not interact but which may contain some aggregate parameters for use in an agent model; a 'passive' environment that does not contain any inherent dynamics but with which agents can interact with variables or structures; and finally an 'active' environment which is dynamic and an active part of the agent model. They propose that an SD model could be used to build the 'active' environment, as described later through examples of hybrid simulation modelling for land use.

Additionally, although dealing with SD-DES hybrid simulations, Chahal and Eldabi [8] describe their design concepts for modelling healthcare governance in the UK's National Health Service. They emphasise the importance of understanding intra-departmental as well as inter-departmental interactions. In essence they propose the use of an SD-DES hybrid design to capture both horizontal and vertical integration issues within this system where different elements of the organisation are represented in either SD or DES modules.

Drawing from a wide range of research domains, a number of examples have been summarised here which echo the view explicitly described by Parunak et al. [37] that 'individual behaviour' and 'equations describing observables' are system elements that could be decomposed into AB and SD modules, respectively, for use in hybrid model design. There is also a clear decomposition where local or spatial conditions are not well represented at an aggregate level, as discussed by Rahmandad [40], Lorenz and Jost [27] and Chahal and Eldabi [8], for example. This aspect of system decomposition is highlighted further in section 6 in a review of work by Homer [21], Schieritz and Milling [44] and He et al. [20].

Cognisant of the interplay between scales of the system, system decomposition may therefore be appropriate, in the context of $S D$ and $A B$ hybrid simulation design concepts, on the basis of:

- individual behaviour;

- equations describing observables; or

- where local (including spatial) conditions are not well represented at a higher level of abstraction.

\subsection{Management of Units and Time}

Further considerations for hybrid model design are consistency of units and appropriate representation of timing. Chahal, Eldabi and Mandal [9] describe types of interactions between models within a hybrid design, in this case comprising SD and DES. They describe 'cyclic' interactions, where the models run separately and information is exchanged between consecutive runs with no interaction during run time; 'parallel' interactions, where the models are run at the same time and information is passed between them; and 'planetary' interactions where the DES model runs for every time-step of the SD model with data exchange taking place every time-step. The first of these interactions seems to correspond to our sequential class, while the second could correspond to either the interfaced or the integrated class. The third type of interaction is clearly in the integrated class. This degree of correspondence suggests that the classification we propose here for hybrid AB-SD simulations might have wider applicability to hybrid simulation modelling in general. 
Examples of time management are also provided by Venkateswaran et al. [54] in their SD-DES hybrid model for production planning. Alvanchi, Lee and AbouRizk [2] consider the need to exchange information only when meaningful, i.e. when something has changed. They also recognise that this concept is a function of how different modelling paradigms represent time.

\subsection{Degrees and Representation of Agency}

In introducing their concept for a "continuum of agency", Schieritz and Milling [44] provide a review of the factors used in published literature to describe an agent. They surmise that there are degrees of agency exhibited whereby an 'agent' will have a range of the properties highlighted within the defined continuum. While there is no universally agreed definition of an agent, Macal and North [28] discuss a number of agent characteristics which they consider essential. These include the notion of an agent being an identifiable, bounded entity, and having the ability to act autonomously and to interact with other agents as well as its environment. Importantly for the relationship between $A B$ and SD models, is the dynamic nature of an agent's state, represented by attributes which can affect its behaviour. The collection of agent states at any given time in an AB model is directly linked to the set of state variables within an associated SD model. These references indicate the need to consider the description of an agent within whichever modelling paradigm is being used.

\section{Suggested Categories of Hybrid AB-SD Simulations}

As outlined in section 3, we have identified three classes of design for hybrid AB-SD simulation based on our literature review. These are shown schematically in Figure 4. Examples of where these design classes have been implemented are described in the following sub-sections for integrated, interfaced and sequential classes, respectively. In all three classes, the system is decomposed in accordance with the findings reported in section 4 with SD or AB modules used to represent different aspects of the system. Whilst, for simplicity, only two modules are illustrated below, the number of modules used will depend on the fidelity required. The integrated class incorporates feedback between modules representing a continuous, fluid process. Modules within the interfaced class may be run in parallel with their outputs combined as required to represent the desired output as a function of time. In the sequential class, one module has to be run first and its output then fed to the next. The feedback arrows between modules in the integrated class and the arrows from modules to output in the interfaced class do not constrain flows to a single point in time. Indeed, the expectation is that such flows will usually take place several times during simulation. Only in the sequential class is the flow restricted to a single time point. 

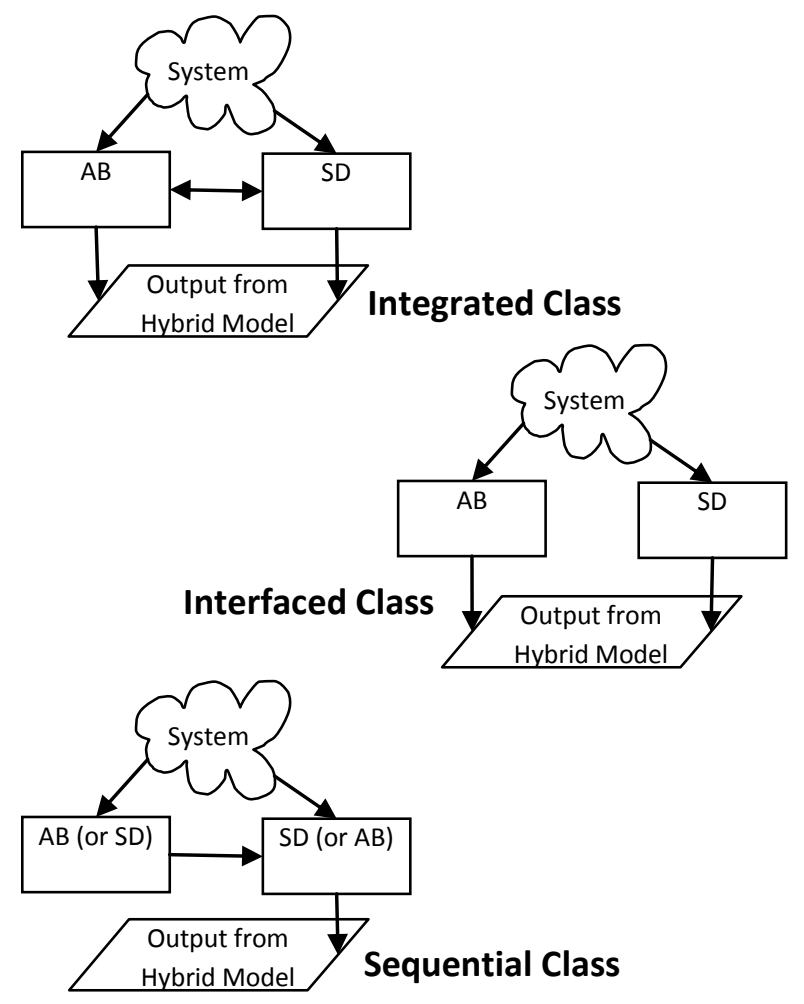

Fig.4. The proposed three classes of hybrid AB-SD simulation.

\subsection{The Integrated Hybrid Design}

Not all of the examples presented here of the AB-SD integrated hybrid design explicitly use different simulation paradigms within the model architecture. Two examples, Sterman [50], and subsequently Sterman and Wittenberg [51], and Akkermans [1], are included here that were implemented entirely in an SD environment but which used $A B$ design principles to derive models that capture the ethos of hybrid AB-SD simulation in order to better represent and analyse complex systems. It is debatable whether such models should be considered truly hybrid or not but we have included them for completeness. It could be argued that they actually form an additional class of agent-oriented SD models.

There are at least three options available to implement the AB-SD integrated hybrid design concept where:

- $\quad$ an SD module is built within agents of an $A B$ module (agents with rich internal structure);

- a level within an SD module is used to bound an aggregate measure of an AB module (stocked agents); or

- an aggregate measure or observation of an $A B$ module is used to influence a parameter within an SD module (parameters with emergent behaviour). 
Examples of each of these implementations are provided below. In the case of agents with rich internal structure and parameters with emergent behaviour, the flow of information between SD and AB modules can be bidirectional. In the case of the stocked agent implementation, however, information flow will tend to be from the $S D$ module to the $A B$ module only, with the net benefit being a representation of local (including spatial) or individual behaviours. It is important to note that the process of feedback between the SD and AB modules within these three interpretations of the integrated design class is not constrained. Feedback is the key feature that provides for an integrated hybrid approach to simulation.

Schieritz and Größler [43] describe an explicit integrated hybrid simulation model for supply chains with SD models representing company decision-making and the links between companies within the supply chain modelled using an $A B$ model. They chose this form of design because the structure, i.e. the interconnected relationships between companies within the supply chain, changes with time and they did not consider this suited to a purely SD representation. They therefore complement the SD approach with $A B$ modelling in order to increase the flexibility of the model to better represent supply chain connectivity as a function of time. In this case, two software packages, namely the Vensim ${ }^{\mathrm{TM}}$ SD package and the eM-Plant ${ }^{\mathrm{TM}}$ or RePast $^{\mathrm{TM}} \mathrm{AB}$ package, were used together in order that the hybrid design could be implemented [18]. Their approach provides a powerful visualisation of the emergent relationships developed in supply chains. It is also worth noting that the implementation of integrated hybrid designs is readily achieved through products such as AnyLogic [4] or, in the case of agent-orientated SD models, through direct coding such as in the Sterman [50] and Akkermans [1] examples that will be described later. Where a spatial dimension is required within a simulation, then hybrid modelling within an AB package such as Repast or Netlogo, for example, or a multi-paradigm package such as AnyLogic, for example, is likely to provide the simplest implementation, avoiding the need for interfacing disparate software.

An illustration of this type of integrated hybrid design, where an SD module is integrated within each agent of an $A B$ module, is provided at Figure 5. In this figure, one of the agents is magnified, revealing its internal structure to be an SD model. In the case of Schieritz and Größler [43], the agents are links between companies. However, the representation in Figure 4 is a generic representation of the concept. 


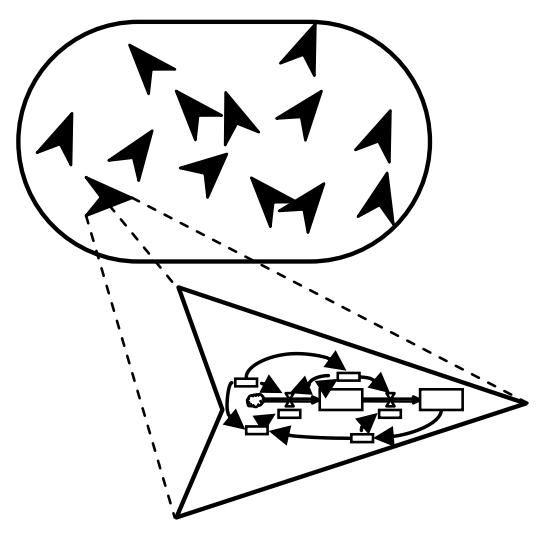

Fig.5. An integrated hybrid design concept representing an implementation of agents with rich internal structure.

The SERD model described by Gaube et al. [17] in a special issue of the Landscape Ecology Journal [33] has a different type of integrated hybrid design. This model has been designed to represent coupled socio-ecological systems in land-change science for a defined region of Austria. It primarily consists of three modules: an $A B$ module representing decision-making by farmsteads, government authorities and 'other important actors' referred to as the ABM; a spatially explicit land use module that represents individual parcels of land and is referred to as the LUM; and an integrated stock and flow socio-ecological module of aggregate Carbon and Nitrogen flows, referred to as the SFM. The potential feedback mechanisms within the model are described as: 'Feedbacks between the ABM and the SFM mostly proceed via the LUM. For example, if farmers decide to change how a parcel of land is used, this affects the area given over to a defined land use (e.g. cattle grazing) and possibly the farming intensity (e.g. amount of fertilizer per hectare and year). Direct feedback between the ABM and SFM modules can also occur. For example, households or companies may switch between fuels (e.g. heating oil or wood) and thereby affect the system's carbon balance' [17, p1152]. The LUM module was implemented using a GIS mapping system and standard commercial database software, whilst the ABM and SFM modules were implemented within the AnyLogic [4] simulation software. The mapping module reflects the spatial results of management decisions made within the $A B$ module for land use allocation. The SERD model therefore represents both the 'stocked agent' and 'parameter with emergent behaviour' flavours of integrated hybrid design where, for example, the implication of net carbon and nitrogen flows impact local decision-making and where the aggregate representation of local decisions impact the system at a higher level of the system hierarchy (or scale), respectively. These forms of implementation are illustrated in Figures 6 and 7. 


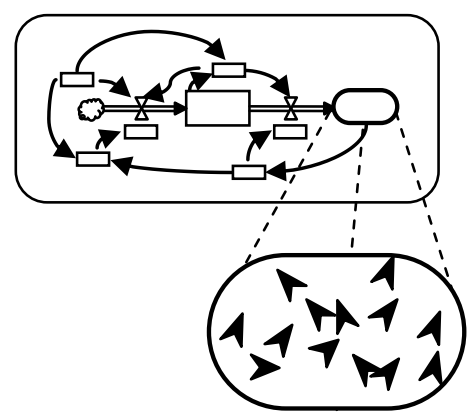

Fig.6. An integrated hybrid design concept representing 'stocked agents'.

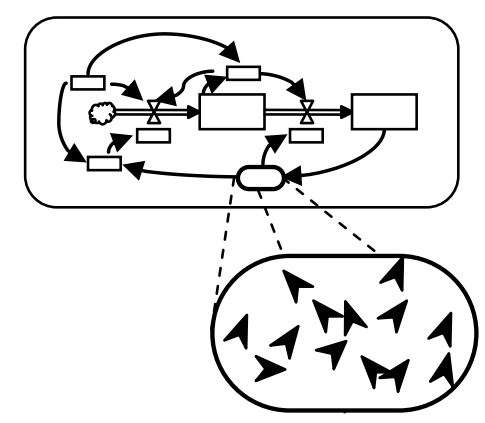

Fig.7. An integrated hybrid design concept representing 'parameters with emergent behaviour'.

The land change science work as reported by Verburg and Overmars [55] can be represented by the 'stocked agent' integrated hybrid design. In this concept, the $A B$ model is bound by an aggregate stock level such as products or land use classification, for example. Within the defined bound of the aggregate stock level, detailed information can be made available through observation and analysis of agent behaviour.

Verburg and Overmars [55] chose a hybrid approach for their Dyna-CLUE model in order to explicitly capture cross-scale coupling between local, regional and national aspects of the system. They considered that aggregate models were unable to adequately capture local land change responses to regional or global demand or policy. On first inspection, the Dyna-CLUE model looks to provide an example of the interfaced hybrid design concept.

However, when the time-stepping nature of the model is considered, there is sustained feedback and so this model is assessed to be an integrated design aligned to the stocked agent implementation as illustrated in Figure 6. The difference between the implementation here by Verburg and Overmars [55] and that reported by He et al. [20] is slight and yet they fall within different hybrid design concepts. In the case of the LUSD model, the national scale of land demand was determined for the 50-year period considered and then the local response to that modelled such that national demand was satisfied. In the case of Verburg and Overmars [55], they iterate between national and local models at each time step.

Kieckhäfer et al. [23] describe their integrated hybrid model which represents product portfolio strategies for European automotive manufacturers responding to regulatory requirements for $\mathrm{CO}_{2}$ emissions of new vehicles 
and availability and price factors associated with crude oil. They present a scenario where manufactures have to use a parallel strategy for manufacturing cars with either conventional or alternative fuel and powertrain technology. Their analysis recognises three classes of actor, namely: manufacturers, legislators and customers, and the dynamics between them. They present an integrated model comprising SD and AB modules with the interaction from SD module to $A B$ module capturing the impact of production costs on car sale price and the influence of fuel price on consumer choice of vehicle technology. The interaction from AB module to SD module captures the impact of product class sales on manufacturing decision making. This model captures the concepts presented in Figures 6 and 7.

Chaim and Streit [10] describe a hybrid design for modelling pension fund governance. Whilst the detailed interactions of the $A B$ and $S D$ models are not described explicitly, individual decision-making of an agent population is modelled using fuzzy logic to represent their decision to participate or not in the pension fund. Demographic factors such as age, income and health are factored into the representation of the agents as well as their ability to interact with government, which is also represented as an agent. An SD model is used to represent the governance of the pension fund using an asset and liabilities model that is influenced by the emergent dynamics of the population. The SD model uses aggregate measures influencing assets and liabilities such as financial contributions made, potential for future financial contributions based on age and salary projections, payments to those retired and future liabilities for payments based on mortality predictions of those retired.

Three examples are now considered that were designed and implemented entirely within an SD model but drew on $A B$ design principles.

Sterman [50] and later Sterman and Wittenberg [51] described a model for the birth, evolution and death of scientific paradigms based on the theories of Kuhn. In this model, the scientific paradigms can be considered as agents with rich internal structure. The level of confidence held in a paradigm is a key property of each agent and determines its potential to attract researchers and hence sustain its existence and standing in the scientific world. While agents are generated randomly with randomly sampled inherent strengths, the rate of agent generation depends on the state of the dominant paradigm. Ultimately, the behaviour of the model can be shown to be driven by a number of interacting feedback loops.

Akkermans [1] models a three-tier decentralised supply chain network where companies, referred to as actors, are the agents within the design. Ten original equipment manufacturers feed an end market (also considered an agent) and are, in turn, supplied by a further two tiers in the supply network. Akkermans' focus for this analysis is on the impact of actors balancing short-term and long-term relationships with suppliers and customers in the network, and represents decision-making within each agent. On reflection, he reports that 'it appears feasible, and even advantageous, to implement $A B$ models within a SD environment' $[1, p 8]$. 
Duggan's [14] 'simulator for continuous agent-based modelling' describes a method for building hybrid models within an SD modelling framework. A case study is provided of a competitive market that includes a social network. In this, three classes of agent within a population of 100 decide whether to align to one of two companies. This example explores the concept of agents with rich internal structure.

Whilst these three examples are not about the explicit combination of different modelling techniques, the structure and thinking behind their design are informative when considering architectures and design concepts at the outset of a study. There is no spatial component in any of these examples although local circumstances and decision-making are captured within the context and structure of a wider adaptive system. Further examples of integrated hybrid designs can be found in SD-DES hybrids such as the two reported examples for production planning by Rabelo et al. [39] and Venkateswaran et al. [54].

The illustration at Figure 8 brings together the examples highlighted above with the three types of integrated design discussed, showing how they all fit within the generic integrated hybrid class. It can be seen that combinations of the three possible implementations can be used to represent scale within the system being modelled. Integrated feedback within these models is also highlighted with the greyed block arrows. 


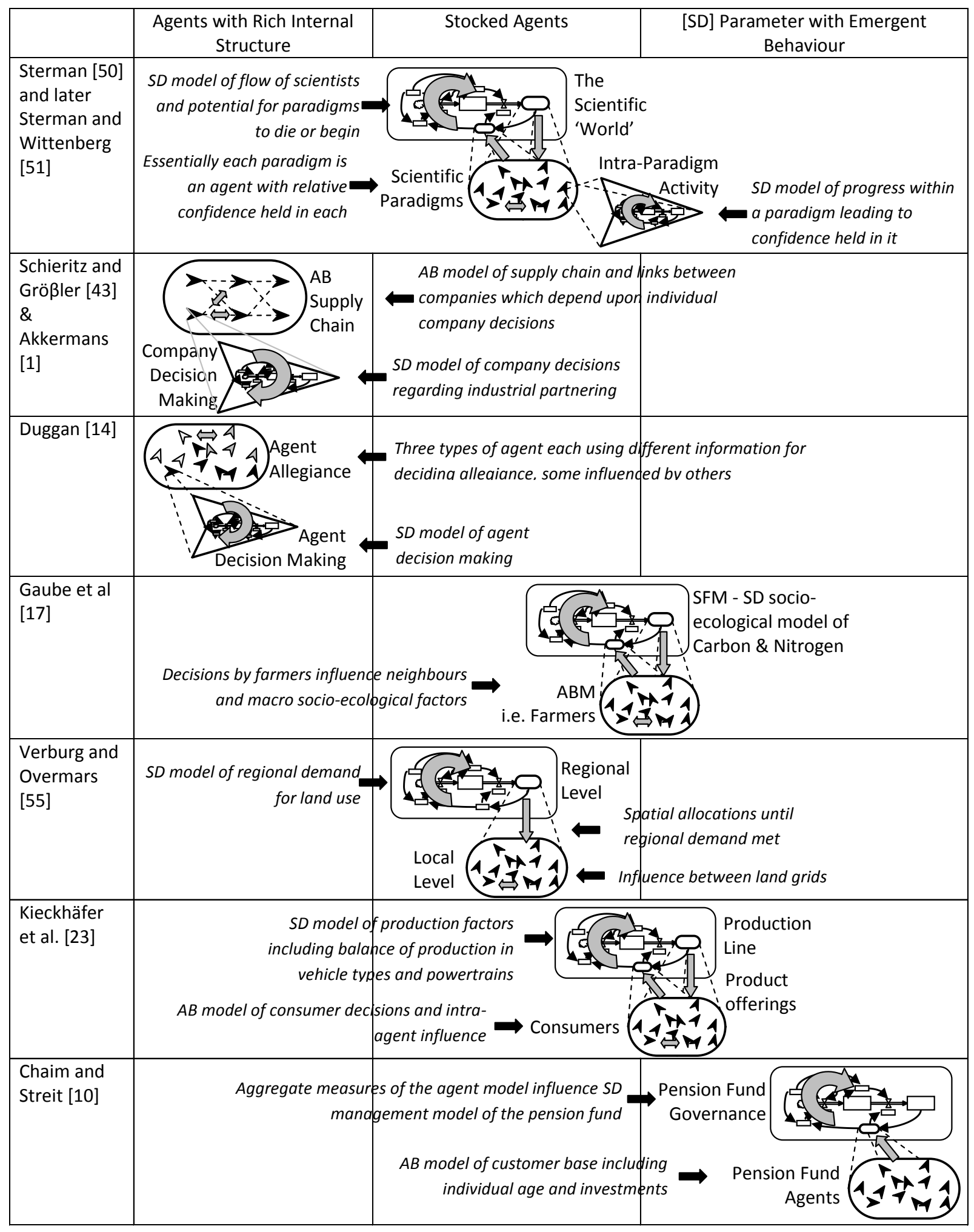

Fig.8. Representation of reviewed models that fall within the integrated hybrid design.

\subsection{The Interfaced Hybrid Design}


Based on our review of the hybrid AB-SD literature, we support the view of Shanthikumar and Sargent [48] that there can be a fine line between what falls within one hybrid design category and another. At the time of writing, no examples comprising SD and $A B$ modules for this design concept have been found in the published literature. However, and whilst not the focus for this review of hybrid AB-SD design classes, Dubiel and Tsimhoni [12] describe their hybrid simulation that comprises $A B$ and DES modules. Within the $A B$ module, an agent looks to travel to a 'goal object', avoiding obstructions and obtaining directions from dynamic information objects (people) or stationary information objects (maps) to get from their starting point to the 'goal'. They can walk all of the way or take a tram for part of the way, the tram being represented within the DES module. If taking the tram, the agent queues at a tram stop and, when a tram arrives, moves out of the AB module and into the DES module. The agent departs from the tram at a stop after a defined period of time, transitioning from the DES module to the $A B$ module also. If the agent gets lost, then they return to their last point of interaction and start their journey again. There is no direct feedback between $A B$ and DES modules, although the journey can be faster if agents learn to take the tram. We therefore consider that this is an example of an interfaced hybrid design as the agent is either walking within the $A B$ module or is travelling within the DES module; i.e. alternating between independent $A B$ and DES modules. The net result of total travel time can, however, require output from both modules.

\subsection{The Sequential Hybrid Design}

A sequential design is where one modelling technique is used to inform the design, use or starting conditions of another. Three examples of this approach covering workforce planning, population dynamics and land use dynamics are presented - Homer [21], Schieritz and Milling [45] and He et al. [20], respectively.

Homer [21] describes an analysis of field service strategy for handling work volumes with a staff of mixed training, preparedness and experience. The primary model is an SD representation of the strategic issues to be addressed by a field service company such as demand, workforce scheduling, service quality, customer satisfaction and finance. Part of this model captures the training of the workforce. If all staff were trained to repair all equipment, then a straightforward aggregate ratio could be used to link time spent on training to time spent on revenue-earning activity. However, the workforce considered, as with any workforce, had a mix of experienced and trained staff. Not all staff could repair all equipment and therefore a detailed analysis was required in order to determine the readiness of the workforce to service demand. With further analysis, it became apparent that a form of empirical relationship, typically represented within SD models as a so-called 'table function', would be required for part of this model but despite a general consensus on the likely shape of the table function, there was no hard data available to confirm this. This was overcome by developing a 'micro-scale' agent-based model to replicate the workforce and using this to determine the nature of the table function required in the SD 'macro' model. The paper describes in detail how this was achieved. In conclusion, Homer reflects that 'SD model 
parameters should be estimated using data below the level of aggregation of model variables wherever possible' [21, p159]. However, it is not always straightforward especially when using lower aggregate historical data for use in an SD model designed to predict future outcomes beyond the range of past experiences. Here the process of developing a micro-level model for service queuing and task allocation to individual engineers in order to develop a key table function for service readiness was considered successful. Homer reflects that while it was time consuming to complete, the process of doing this produced greater insight into the issues around cross-training, and achieved much more stakeholder buy-in than if the table function had been based purely on assumption or judgement.

Schieritz and Milling [45] report the use of $A B$ simulation to identify and quantify macro structures that can be used within an SD model. The main objective of their paper is to assess the potential to use AB modelling where either the structure of a system is unknown to decision makers working within that system or their collective mental model cannot be captured. They state that where either condition cannot be fulfilled, then 'it would be difficult to quantify an SD model or to even identify the causal problem structure' of the system. They begin by using a relatively simple SD population model as an example in which the level of population size is regulated by the rates of reproduction and death, the reproduction rate being a function of the population level and fertility. In turn, fertility is a function of the availability of resources. They observe, however, that fertility depends upon local conditions and that the SD model can only represent global availability of resources.

They therefore build an $A B$ model of a population of agents which can be configured so that all of the agents are either static or mobile. In the static case, the agents deplete their locally available resources and hence their fertility is reduced, which in turn slows the rate of population growth. However, in the mobile case the agents can access resources and thereby maintain fertility levels. In this case, the population grows more rapidly than in the static case and reaches a stable population level where the rates of reproduction and death are in balance. A plot of average fertility versus availability of resources reveals two different relationships that could be used via a table function within an SD model.

Schieritz and Milling [45] then go on to extend their analysis of population dynamics to the situation where the causal relationships of the SD model are not known. Here they investigate the use of an $A B$ approach to determine the design of the SD model. However, they state that one prerequisite to this approach is that the policies of the individual agents have to be identifiable. They also note that an advantage of the parallel or sequential use of different methodologies for the analysis of a single problem is the ability to validate results, although they concede that this may require a substantial effort.

In reporting on land use dynamics in China, He et al. [20] reports on the Land Use Scenario Dynamics (LUSD) model which incorporates SD and CA modules. Within this design concept, the SD module firstly determines 
national and regional demand for land use based on factors such as land policy, demographics, market demand, the economy, and influence of technology. The CA module then provides a spatial representation of local land allocation to meet the aggregate demand defined by the SD module and considers local factors such as land suitability, land inheritance and the influence of neighbours. The output from the LUSD model is provided by the CA module and is in the form of a geographic map on which land change is plotted.

Generic representations of the sequential design are presented in Figure 9, illustrating the design concepts described by the examples above.

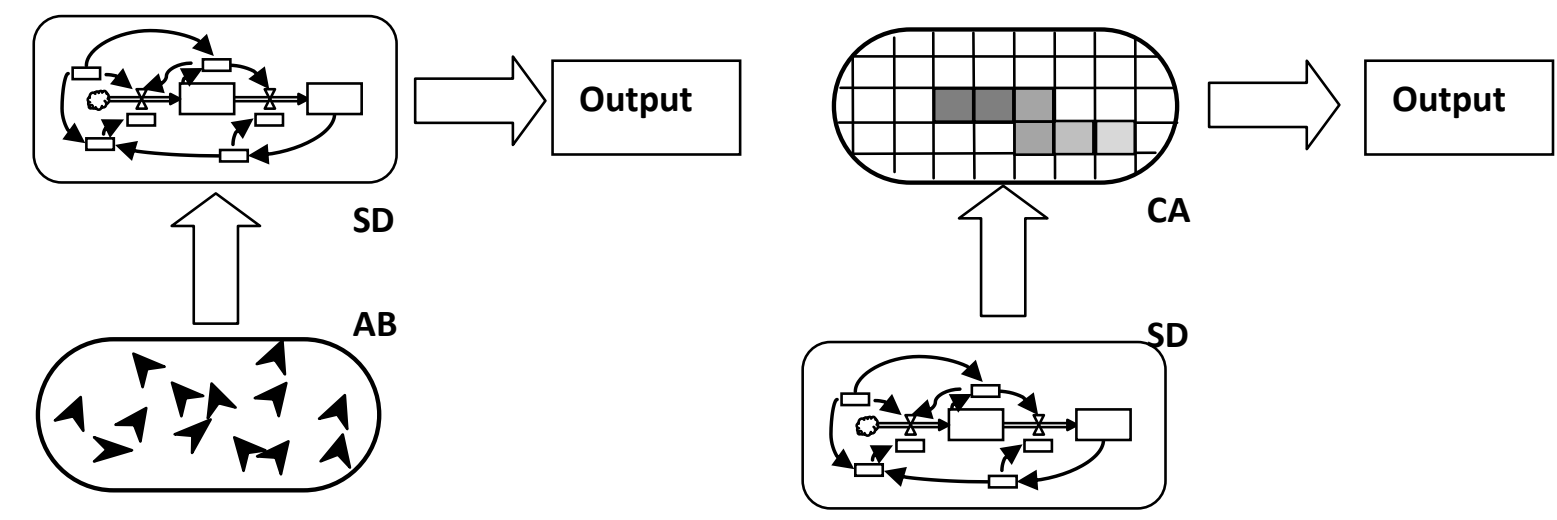

Fig.9. Sequential hybrid design concepts using an $A B$ module to inform an SD module and an SD module to inform a CA module.

\section{Modelling System Hierarchy}

One of the key factors arising from the review of hybrid simulation modelling is the desire to represent interdependencies between different levels of a system's hierarchy or scale. Verburg and Overmars [55, p1177], for example, reflect that the implementation of a hybrid model provided for a 'step towards better addressing cross-scale interactions in land use modelling'. We suggest that it is in the representation of the coupling between these scales that hybrid approaches should be able to demonstrate benefits beyond the application of individual paradigm approaches.

In presenting a review of simulation modelling tools and techniques, Borshchev and Filippov [7] describe the notion of the level of abstraction in regards to the appropriate application of simulation paradigms. However, abstraction in terms of coupled sub-systems has a relative as well as an absolute meaning. For example, $A B$ is good at representing the decision-making of individual employees, but may also be used to represent the decision-making of individual companies or indeed of individual nations. The level of abstraction therefore has to be placed within a context of system hierarchy when considering hybrid model design, as illustrated in Figure 10. Here, two examples of hybrid simulation design are represented comprising two or three modules. The two- 
module design incorporates an SD module to represent aspects of the system at higher levels of scale than in the $A B$ module. For the three-module design example shown here, separate $A B$ modules would represent both the highest and lowest layers of system scale while an SD module would represents an intermediate layer.
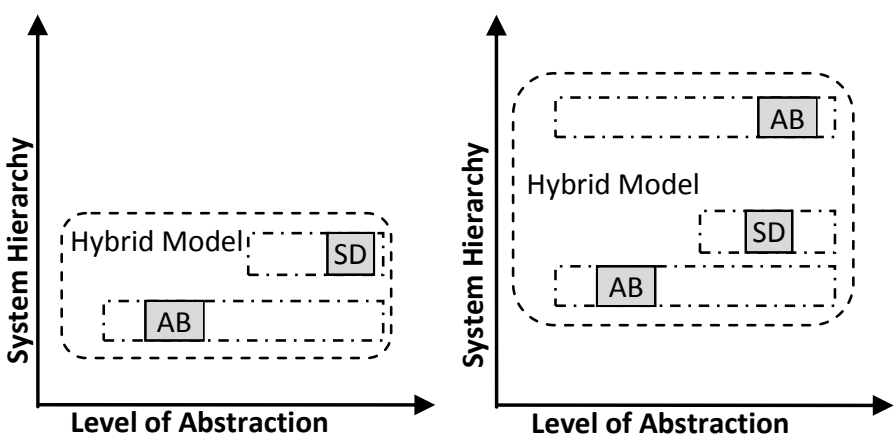

Fig.10. Level of abstraction in the context of system hierarchy and hybrid simulation constructs.

Throughout this paper, SD and AB modules have been used to illustrate hybrid design concepts. However, there is a range of modelling tools available today with which to represent various aspects of a system and from which to design appropriate architectures for hybrid models. Some variations of the sub-categories 'rich internal structure' and 'parameters with emergent behaviour' of the integrated hybrid design class are illustrated in Figure 11 as examples.

SD parameter with emergent behaviour
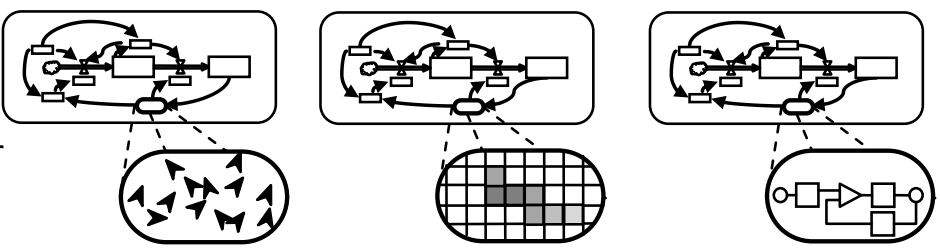

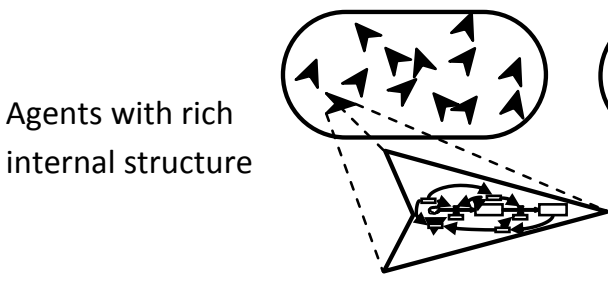

SD

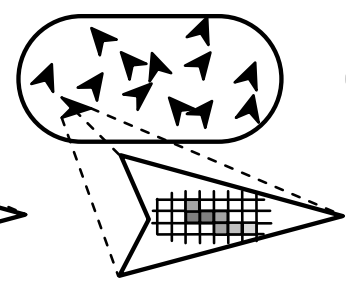

Cellular Automata

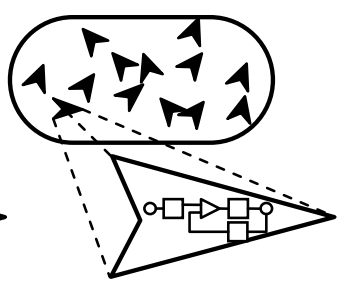

Discrete Event

Fig.11. Examples of integrated hybrid designs comprising modules from different modelling paradigms.

A further extension to the design concepts defined thus far is to consider time or event driven reconfiguration of a hybrid model. This concept is illustrated in Figure 12 for an event trigger switching in or out the 'parameters with emergent behaviour' sub-category of the integrated hybrid design. This concept reflects the potential for optimal modelling where the 'nature' of the system changes over time or at specified events. For example, in considering the diffusion of innovations, including high-tech products, Rogers [41] describes how population demographics and social interactions are significant until a critical aggregate condition for product adoption has 
been achieved. Thereafter, a wholly aggregated model is sufficient to represent what becomes a 'self-sustaining' diffusion process [41, pp343-357].

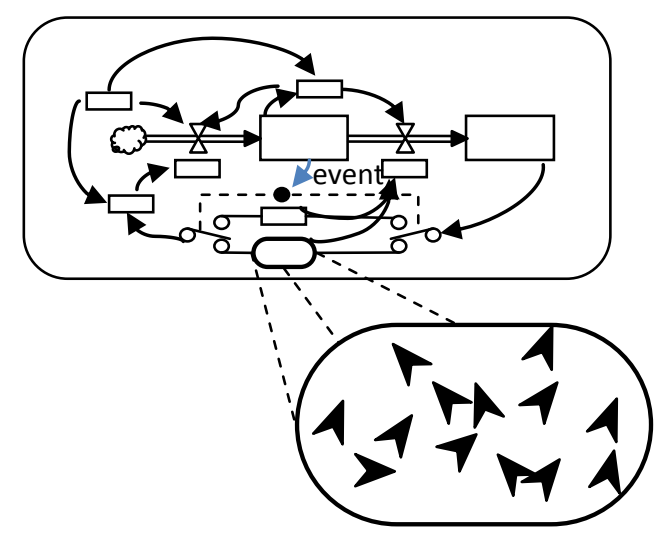

Fig.12. Event reconfigurable design optimisation concept.

This switching concept is further described in the work of Bobashev et al. [6] which explores the potential for using thresholds to switch between $A B$ and SD models. The model described represents cities linked through air travel in the context of modelling epidemics. The concept proposed originates from the premise that when there are many active agents, the law of large numbers and central limit theorem could be applicable. They observe that such a modelling approach would be more computationally efficient, especially where the number of agents being modelled is very large. The paper also introduces the concept of dynamic threshold switching for each city, whereby the $A B$ or SD representation is switched in or out depending upon the number of local agents (in that city) that are 'active', i.e. exposed or infectious. Levin and Levin [26] explore the potential for Finite State Machines (FSM) to switch between modes, which are represented by SD modules, of a continuous system. They provide an illustrated example of an FSM controlling modes within an SD model which represents the controlled volume and water temperature of a bath. This model is wholly constructed within the SD modelling environment. There are four discrete modes within the SD model controlled by the FSM, representing heating control (ON or OFF) and drain control (OPEN or CLOSED).

Switching may also be of use during model initialization at the start of a simulation. For example, Rahmandad [40] observes that the specific social network structure strongly conditions the probability of an epidemic taking off in comparing SD and AB models. This concurs with Rogers' earlier work, discussed above.

\section{Conclusions}

The growing interest in hybrid simulation approaches may be partly explained by improvements in simulation education and by the increasingly complex nature of problems being faced by OR professionals. Many such problems involve interacting elements of a different nature. Where these elements are best represented by 
different simulation paradigms, then modellers face the choice of trying to identify the best single paradigm and applying it to the whole system, or adopting multiple paradigms, i.e. a hybrid simulation approach. Once a hybrid approach has been selected, however, there are still choices to be made regarding its nature. In this paper, we have sought to identify some broad classes of hybrid simulation, drawing widely on the published literature. Borrowing heavily from Shantikumar and Sargent's [48] classification of hybrid modelling types, we have revised and adapted this classification for hybrid simulations involving agent-based simulation and system dynamics. However, we believe that this classification may be more widely applicable to other forms of hybrid simulation.

We have discussed the notions of integrated, interfaced and sequential hybrid simulation designs. The class of integrated designs is particularly prominent and varied. We have identified several variants of this design, including a variant developed entirely within the SD paradigm but employing concepts aligned with agent-based modelling. Whether this variant can actually be considered a hybrid simulation at all, or whether it is better referred to as an agent-oriented SD model is open to discussion but we have included this variant here for completeness.

Despite the growing literature and interest in hybrid simulation modelling, there still appears to be very little work which attempts to classify or organize the various types of hybrid simulations. This paper is intended to be a step in that direction. Lorenz and Jost [27] proposed that the discussion and selection of suitable modelling methodologies should be a key factor in the early stages of the modelling process. It is hoped that the classification suggested here will aid analysts and researchers in having that discussion when considering simulation model design concepts prior to undertaking detailed model development. Furthermore, while the potential for AB-SD hybrid models to capture the nature of cross-scale system dynamics is a distinct advantage yet to be fully exploited, very little has been reported about the benefits of adopting such approaches and this remains an area where much more research is needed.

\section{Acknowledgements}

We would like to thank the two anonymous referees for their helpful and constructive comments which have helped to improve the clarity and content of this paper. We also thank Robert Sargent for his helpful and encouraging comments on an earlier draft.

\section{References}

[1] Akkermans H (2001). Emergent supply networks: system dynamics simulation of adaptive supply agents, Proceedings of the 34th Annual Hawaii International Conference on System Sciences.

[2] Alvanchi A, Lee SH and AbouRizk SM (2009). Meaningful level of change in hybrid simulation for construction analysis. Proceedings of the 2009 Winter Simulation Conference. 
[3] Alvarez AHR, Solis D, Cano SAR and Sala-Diakanda SN (2006). System dynamics simulation of the expansion of the Panama Canal. Proceedings of the 2006 Winter Simulation Conference.

[4] Anylogic [URL: http://www.xjtek.com accessed 13 September 2010]

[5] Argonne National Laboratory [URL: http://www.dis.anl.gov/exp/cas/index.html accessed 9 September 2010].

[6] Bobashev GV, Goedecke DM, Yu F and Epstein JM (2007). A hybrid epidemic model: combining the advantages of agent-based and equation-based approaches. Proceedings of the 2007 Winter Simulation Conference.

[7] Borshchev A and Filippov A (2004). From system dynamics and discrete event to practical agent based modeling: reasons, techniques, tools. Proceedings of the 22nd International Conference of the System Dynamics Society.

[8] Chahal K and Eldabi T (2008). Applicability of hybrid simulation to different modes of governance in UK healthcare. Proceedings of the 2008 Winter Simulation Conference.

[9] Chahal K, Eldabi T and Mandal A (2009). Understanding the impact of whiteboard on A\&E department operations using hybrid simulation. Proceedings of the 27th International Conference of the System Dynamics Society.

[10] Chaim RM and Streit RE (2008). Pension funds governance: combining SD, agent based modelling and fuzzy logic to address dynamic asset and liability management (ALM) problem. Proceedings of the 26th International Conference of the System Dynamics Society.

[11] Demirel G (2006). Aggregated and disaggregated modeling approaches to multiple agent dynamics. Proceedings of the 24th International Conference of the System Dynamics Society.

[12] Dubiel B and Tsimhoni O (2005). Integrating agent based modeling into a discrete event simulation. Proceedings of the 2005 Winter Simulation Conference, pp $1029-1037$.

[13] Duggan J (2008a). Equation-based policy optimization for agent-oriented system dynamics models. System Dynamics Review 24, 97-118.

[14] Duggan J (2008b). A simulator for continuous agent-based modelling. Proceedings of the 26th International Conference of the System Dynamics Society.

[15] Forrester JW (1961). Industrial Dynamics. MIT Press, Cambridge, MA. 
[16] Garro A and Russo W (2010). easyABMS: a domain-expert oriented methodology for agent-based modeling and simulation. Simulation Modelling Practice and Theory 18, 1453-1467.

[17] Gaube V et al (2009). Combining agent-based and stock-flow modelling approaches in a participative analysis of the integrated land system in Reichraming, Austria. Landscape Ecol 24, 1149-1165.

[18] Größler A, Stotz M and Schieritz N (2003). A software interface between system dynamics and agent based simulations - linking Vensim ${ }^{\circledR}$ and RePast ${ }^{\circledR}$. Proceedings of the $21^{\text {st }}$ International Conference of the System Dynamics Society.

[19] Gupta UG (1997). Using citation analysis to explore the intellectual base, knowledge dissemination and research impact of Interfaces (1970-1992). Interfaces 27, 85-101.

[20] He C, Pan Y, Shi P, Li X, Chen J, Li Y and Li J (2004). Developing land use scenario dynamics model by the integration of system dynamics model and cellular automata model. Proceedings of the Geoscience and Remote Sensing Symposium 2004, pp 2647-2650.

[21] Homer JB (1999). Macro- and micro-modelling of field service dynamics. System Dynamics Review 15, 139-162.

[22] Jahangirian M, Eldabi T, Naseer A, Stergioulas LK and Young T (2010). Simulation in manufacturing and business: a review. European Journal of Operational Research 203, 1-13.

[23] Kieckhäfer K, Walther G, Axmann J and Spengler T (2009). Integrating agent-based simulation and system dynamics to support product strategy decisions in the automotive industry. Proceedings of the 2009 Winter Simulation Conference, pp 1433-1443.

[24] Lane DC (1994). With a little help from our friends: how system dynamics and soft OR can learn from each other. System Dynamics Review 10, 101-134.

[25] Lane DC and Husemann E (2008). Steering without Circe: attending to reinforcing loops in social systems. System Dynamics Review 24, 37-61.

[26] Levin T and Levin I (2003). Integrating hybrid modeling with system dynamics. Proceedings of the 21st International Conference of the System Dynamics Society.

[27] Lorenz T and Jost A (2006). Towards an orientation framework in multi-paradigm modeling. Proceedings of the 24th International Conference of the System Dynamics Society.

[28] Macal CM and North MJ (2010). Tutorial on agent-based modelling and simulation. Journal of Simulation 4, 151-162. 
[29] Macal C (2010). To agent-based simulation from system dynamics. Proceedings of the 2010 Winter Simulation Conference. B. Johansson, S. Jain, J. Montoya-Torres, J. Hugan, and E. Yücesan, eds., (December).

[30] Marin M, et al (2006). System dynamics and agent-based simulations for workforce climate. Proceedings of the 2006 Winter Simulation Conference, pp 667-71.

[31] Martinez-Moyano IJ, Sallach DL, Bragen MJ and Thimmapuram PR (2007). Design for a multilayer model of financial stability: exploring the integration of system dynamics and agent-based models. Proceedings of the 25th International Conference of the System Dynamics Society.

[32] McCarthy IP, Tsinopoulos C, Allen P and Rose-Anderssen C (2006). New product development as a complex adaptive system of decisions. Journal of Product Innovation Management 23, 437-456.

[33] Milne E, Aspinall R and Veldkamp T (editors) (2009). Journal Landscape Ecology Special Issue: Integrated modelling of natural and social systems in land change science. Landscape Ecol (2009) 24, 1145-1270.

[34] Morecroft JDW and Robinson S (2005). Explaining puzzling dynamics: comparing the use of system dynamics and discrete-event simulation. Proceedings of the 23rd International Conference of the System Dynamics Society.

[35] Netlogo [URL: http://ccl.northwestern.edu/netlogo accessed 13 September 2010]

[36] North MJ and Macal CM (2009). Agent-based modeling and systems dynamics model reproduction. International Journal of Simulation Process Modeling 5, 256-271.

[37] Parunak HV, Savit R, and Riolo RL (1998). Agent-Based Modelling Vs. Equation-Based Modelling: A Case Study and Users ' Guide. Proceedings of Workshop on Modeling Agent-Based Systems, Paris. SpringerVerlag, Berlin, pp10-25.

[38] Phelan SE (1999). A note on the correspondence between complexity and systems theory. Systemic Practice and Action Research 12, 237-246.

[39] Rabelo L, Helal M, Son YJ, Jones A, Min J and Deshmukh A (2003). A hybrid approach to manufacturing enterprise simulation. Proceedings of the 2003 Winter Simulation Conference.

[40] Rahmandad H (2004). Heterogeneity and network structure in the dynamics of contagion: comparing agent-based and differential equation models. Proceedings of the 22 nd International Conference of the System Dynamics Society.

[41] Rogers EM (2003). Diffusion of Innovations ( $5^{\text {th }}$ edition). Free Press, New York. 
[42] Sargent RG (1994). A historical view of hybrid simulation / analytic models. Proceedings of the 1994 Winter Simulation Conference, WSC 04, pp 383-386.

[43] Schieritz N and Größler A (2003). Emergent structures in supply chains - a study integrating agent-based and system dynamics modelling. System Sciences, 2003. Proceedings of the 36th Annual Hawaii International Conference on System Sciences.

[44] Schieritz $\mathrm{N}$ and Milling PM (2003). Modeling the forest or modeling the trees. Proceedings of the $21^{\text {st }}$ International Conference of the System Dynamics Society.

[45] Schieritz N and Milling PM (2009). Agents first! Using agent-based simulation to identify and quantify macro structures. In Qudrat-Ullah, H, Spector MJ and Davidsen PI (eds), Complex Decision Making Theory and Practice. Springer: London, pp 139-152.

[46] Scholl HJ (2001a). Agent-based and system dynamics modeling: a call for cross study and joint research. Proceedings of the 34th Annual Hawaii International Conference on System Sciences.

[47] Scholl HJ (2001b). Looking across the fence: comparing findings from SD modeling efforts with those of other modelling techniques. Proceedings of the 19th International Conference of the System Dynamics Society.

[48] Shanthikumar JG and Sargent RG (1983). A unifying view of hybrid simulation / analytic models and modeling. Operations Research 31, 1030-1051.

[49] Stella [URL: http://www.iseesystems.com accessed 13 September 2010]

[50] Sterman JD (1985). The growth of knowledge: testing a theory of scientific revolutions with a formal model. Technological Forecasting and Social Change 28, 93-122.

[51] Sterman JD and Wittenberg J (1999). Path dependence, competition and succession in the dynamics of scientific revolution. Organization Science 10, 322-341.

[52] Tako AA and Robinson S (2010). Model development in discrete-event simulation and system dynamics: an empirical study of expert modellers. European Journal of Operational Research 207, 784-794.

[53] Thomsen JS, Mosekilde E and Sterman JD (1991). Hyperchaotic phenomena in dynamic decision making. In Singh MG and Trave-Massuyes L (Eds.): Decision Support Systems and Qualitative Reasoning, Elsevier Science Publishers, Amsterdam, The Netherlands, pp.149-154.

[54] Venkateswaran J, Son YJ and Jones A (2004). Hierarchical production planning using a hybrid system dynamic-discrete event simulation architecture. Proceedings of the 2004 Winter Simulation Conference. 
[55] Verburg PH and Overmars KP (2009). Combining top-down and bottom-up dynamics in land use modeling: exploring the future of abandoned farmlands in Europe with the Dyna-CLUE model. Landscape Ecol 24, 1167-1181.

[56] Wakeland WW, Gallaher EJ, Macovsky LM and Aktipis CA (2004). A comparison of system dynamics and agent-based simulation applied to the study of cellular receptor dynamics. Proceedings of the 37th Annual Hawaii International Conference on System Sciences. 